Pacific

Journal of

Mathematics

TRANSVERSAL HOLOMORPHIC SECTIONS AND

LOCALIZATION OF ANALYTIC TORSIONS

Huitao Feng AND XiaOnan Ma

Volume $219 \quad$ No. 2

April 2005 


\title{
TRANSVERSAL HOLOMORPHIC SECTIONS AND LOCALIZATION OF ANALYTIC TORSIONS
}

\author{
Huitao Feng And XiaOnAn Ma
}

\begin{abstract}
We prove a Bott-type residue formula twisted by $\wedge\left(\mathbb{V}^{*}\right)$ with a holomorphic vector bundle $\mathbb{V}$, and relate certain analytic torsions on the total manifold to the analytic torsions on the zero set of a holomorphic section of $\mathbb{V}$.
\end{abstract}

\section{Introduction}

Beasley and Witten [2003], studying half-linear models, have described a compactification on any Calabi-Yau threefold $Y$ that is a complete intersection in a compact toric variety $X$. In particular, a remarkable cancellation involving the instanton effect [Beasley and Witten 2003, (1.3)], involving certain determinants of the $\bar{\partial}$-operator, was derived directly from a residue theorem. One would like to understand its implications in mathematics, for example in Gromov-Witten theory. Bershadsky, Cecotti, Ooguri and Vafa [Bershadsky et al. 1993; 1994] predicted that the analytic torsion of Ray-Singer will play a role regarding the genus-1 GromovWitten invariant. Thus we naturally try to understand the results about analytic torsion first.

As an application of [Bismut and Lebeau 1991] and the localization formula (1-3) in this paper, we were able to relate certain analytic torsions on the total manifold with the zero set of a holomorphic transversal section of $\mathbb{V}$, generalizing [Bismut 2004, Theorem 6.6] and [Zhang n.d.] with $\mathbb{V}=T X$ therein. We expect our formula will be useful for understanding [Beasley and Witten 2003, (1.3)] from a mathematical point of view.

This paper is organized as follows. In Section 1 we prove a Bott-type residue formula. In Section 2 we get a localization formula for Quillen metrics. In Section 3 we get a localization formula for analytic torsions under extra conditions. In Section 4, for the reader's convenience, we write down six intermediate results, corresponding to [Bismut and Lebeau 1991, Theorems 6.4-6.9].

MSC2000: 58J52, 32L10, 58J20, 32C35, 57R20.

Keywords: analytic torsion, characteristic classes, characteristic numbers, residue formula.

Feng was partially supported by the NNSF of China (10271059). 


\section{A Bott-type residue formula}

In this section, along the lines of [Bismut 1986, §1], we give a Bott-type residue formula (1-3) by assuming that the holomorphic section is transversal; compare to [Beasley and Witten 2003, (2.32), (2.34)].

Let $X$ be a compact complex manifold with $\operatorname{dim} X=n$ and let $\mathbb{V}$ be a holomorphic vector bundle on $X$ with $\operatorname{dim} \mathbb{V}=l$. We assume that the line bundles $\operatorname{det} T X$ and det $\mathbb{V}$ are holomorphically isomorphic. We fix a holomorphic isomorphism $\phi: \operatorname{det} \mathbb{V}^{*} \simeq \operatorname{det} T^{*} X$, which is clearly unique up to a constant. Thus $\phi$ defines a map from the $\mathbb{Z}_{2}$-graded tensor product $\wedge\left(\overline{T^{*} X}\right) \widehat{\otimes} \wedge\left(\mathbb{V}^{*}\right)$ to $\wedge\left(\overline{T^{*} X}\right) \widehat{\otimes} \wedge^{\max }\left(T^{*} X\right) \subset$ $\wedge\left(T_{\mathbb{R}}^{*} X\right) \otimes_{\mathbb{R}} \mathbb{C}$. We can define the integral of an element $\alpha$ of $\Omega\left(X, \wedge\left(\mathbb{V}^{*}\right)\right)$, the set of smooth sections of $\wedge\left(\overline{T^{*} X}\right) \widehat{\otimes} \wedge\left(\mathbb{V}^{*}\right)$ on $X$, by

$$
\int_{X} \alpha=\int_{X} \phi(\alpha)
$$

Let $v$ be a holomorphic section of $\mathbb{V}$ on $X$. Assume that $v$ vanishes on a complex manifold $Y \subset X$. Then $\left.\nabla v\right|_{Y}:\left.\left.T X\right|_{Y} \rightarrow \mathbb{V}\right|_{Y}$ mapping $U$ to $\nabla_{U} v$ does not depend on the choice of a connection $\nabla$ on $\mathbb{V}$, and $\left.\nabla_{U} v\right|_{Y}=0$ for $U \in T Y$. Let $N$ be the normal bundle to $Y$ in $X$. Assume also that $\left.\nabla v\right|_{Y}:\left.N \rightarrow \mathbb{V}\right|_{Y}$ is injective, and there is a holomorphic vector subbundle $\mathbb{V}_{1}$ on $Y$ such that

$$
\left.\mathbb{V}\right|_{Y}=\left.\mathbb{V}_{1} \oplus \operatorname{Im} \nabla v\right|_{Y}
$$

Let $P^{\mathbb{V}_{1}}$ and $P^{\operatorname{Im} \nabla v}$ be the natural projections from $\mathbb{V}$ onto $\mathbb{V}_{1}$ and $\left.\operatorname{Im} \nabla v\right|_{Y}$.

Let $i(v)$ be the standard contraction operator acting on $\wedge\left(\mathbb{V}^{*}\right)$. A natural question, posed in [Beasley and Witten 2003, §2], is how to express $\int_{X} \alpha$ using the local data near the zero set $Y$ of $v$ for a $\left(\bar{\partial}^{X}+i(v)\right)$-closed form $\alpha$, that is, a form satisfying $\left(\bar{\partial}^{X}+i(v)\right) \alpha=0$.

First we recall an idea due to Bismut [Bismut 1986]; see also [Zhang 1990].

Proposition 1.1. Let $\alpha \in \Omega\left(X, \wedge\left(\mathbb{V}^{*}\right)\right)$ be a $\left(\bar{\partial}^{X}+i(v)\right)$-closed form. Then

$$
\int_{X} \alpha=\int_{X} e^{-\left(\bar{\partial}^{X}+i(v)\right) \omega / t} \alpha \quad \text { for any } \omega \in \Omega\left(X, \wedge\left(\mathbb{V}^{*}\right)\right) \text { and } t>0 .
$$

Proof. For any $\omega \in \Omega\left(X, \wedge\left(\mathbb{V}^{*}\right)\right)$

$$
\int_{X} \bar{\partial}^{X} \omega=\int_{X} \phi\left(\bar{\partial}^{X} \omega\right)=\int_{X} \bar{\partial}^{X} \phi(\omega)=\int_{X} d \phi(\omega)=0 .
$$

From $\left(\bar{\partial}^{X}+i(v)\right)^{2}=0$ and $\left(\bar{\partial}^{X}+i(v)\right) \alpha=0$, we have

$$
\frac{\partial}{\partial s} \int_{X} e^{-s\left(\bar{\partial}^{X}+i(v)\right) \omega} \alpha=-\int_{X}\left(\bar{\partial}^{X}+i(v)\right)\left(\omega e^{-s\left(\bar{\partial}^{X}+i(v)\right) \omega} \alpha\right)=0,
$$

and the desired equality follows. 
Recall that $\left.\nabla v\right|_{Y}:\left.N \rightarrow \operatorname{Im} \nabla v\right|_{Y}$ is an isomorphism that induces isomorphisms of holomorphic line bundles $\phi_{N}=\left(\left.\operatorname{det} \nabla v\right|_{Y}\right)^{*}: \operatorname{det}\left(\left.\operatorname{Im} \nabla v\right|_{Y}\right)^{*} \rightarrow \operatorname{det} N^{*}$ and $\phi_{Y}=\left.\phi\right|_{Y} /\left(\left(\left.\operatorname{det} \nabla v\right|_{Y}\right)^{*}\right): \operatorname{det} \mathbb{V}_{1}^{*} \rightarrow \operatorname{det} T^{*} Y$. These two isomorphisms make the integral $\int_{N}$ along the normal bundle $N$ and $\int_{Y}$ well defined.

Let $h^{\mathbb{V}}$ be a Hermitian metric on $\mathbb{V}$ such that $\mathbb{V}_{1}$ and $\left.\operatorname{Im} \nabla v\right|_{Y}$ are orthogonal on $Y$. Let $g_{1}^{N}$ be a Hermitian metric on $N$ such that $\left.\nabla \cdot v\right|_{Y}:\left.N \rightarrow \operatorname{Im} \nabla v\right|_{Y}$ is an isometry. Let $R^{\mathbb{V}}$ be the curvature of the holomorphic Hermitian connection $\nabla^{\mathbb{V}}$ on $\left(\mathbb{V}, h^{\mathbb{V}}\right)$. Let $j: Y \rightarrow X$ be the natural embedding, and $\left\{Y_{j}\right\}_{j}$ the connected components of $Y$. On $Y$, define

$$
R_{v}^{\mathbb{V}}=-(\nabla \cdot v)^{-1} P^{\operatorname{Im} \nabla v} R^{\mathbb{V}}\left(\cdot, j_{*} \cdot\right) P^{\mathbb{V}_{1}} \cdot \in \overline{T^{*} Y} \widehat{\otimes} \mathbb{V}_{1}^{*} \otimes \operatorname{End} N .
$$

$R_{v}^{\mathbb{V}}$ is well defined since $P^{\operatorname{Im} \nabla v} R^{\mathbb{V}}\left(j_{*} \cdot, j_{*}\right) P^{\mathbb{V}_{1}}=0$. Thus, for $U \in T Y, W \in \mathbb{V}_{1}$, $u_{1}, u_{2} \in N$,

$$
\left\langle R_{v}^{\mathbb{V}}(\bar{U}, W) u_{1}, u_{2}\right\rangle_{g_{1}^{N}}=-\left\langle R^{\mathbb{V}}\left(u_{1}, \bar{U}\right) W, \nabla_{u_{2}} v\right\rangle=\left\langle W, R^{\mathbb{V}}\left(\overline{u_{1}}, U\right) \nabla_{u_{2}} v\right\rangle .
$$

Certainly $\operatorname{det}_{N}\left(\left(1+R_{v}^{\mathbb{V}}\right) / 2 \pi i\right)$ is $\bar{\partial}^{Y}$-closed.

The following result verifies a formula of Beasley and Whitney [2003, (2.32), (2.34)] and generalizes corresponding results in [Zhang 1990], [Liu 1995] and [Bott 1967].

Theorem 1.2. For any $\left(\bar{\partial}^{X}+i(v)\right)$-closed form $\alpha \in \Omega\left(X, \wedge\left(\mathbb{V}^{*}\right)\right)$,

$$
\int_{X} \alpha=\sum_{j} \int_{Y_{j}} \frac{(-1)^{(l-n)\left(n-\operatorname{dim} Y_{j}\right)} \alpha}{\operatorname{det}_{N}\left(\left(1+R_{v}^{\mathbb{V}}\right) /(-2 \pi i)\right)}
$$

Proof. Set

$$
S=\langle\cdot, v\rangle_{h^{\vee}} \in C^{\infty}\left(X, \mathbb{V}^{*}\right) .
$$

By Proposition 1.1, for any $t \in] 0,+\infty[$,

$$
\int_{X} \alpha=\int_{X} e^{-\frac{1}{2 t}\left(\bar{\partial}^{X}+i(v)\right) S} \alpha=\int_{X} e^{-\frac{1}{2 t}\left(\bar{\partial}^{X} S+|v|^{2}\right)} \alpha .
$$

Thus, as $t \rightarrow 0$, the integral $\int_{X} \alpha$ is asymptotically equal to $\int_{{ }_{U}} e^{-\frac{1}{2 t}\left(\bar{\partial}^{X} S+|v|^{2}\right)} \alpha$ for any neighborhood $U$ of $Y$.

Take $y \in Y$. Since $Y$ is a complex submanifold, we can find holomorphic coordinates $\left\{z_{i}\right\}_{i=1}^{n}$ of a neighborhood $U$ of $y$ such that $y$ corresponds to 0 and $\left\{\left(\partial / \partial z_{i}\right)(0)\right\}_{i=m+1}^{n}$ is an orthonormal basis of $\left(N, g_{1}^{N}\right)$, and, moreover,

$$
U \cap Y=\left\{p \in U, z_{m+1}(p)=\cdots=z_{n}(p)=0\right\} .
$$

Let $\left\{\mu_{k}\right\}_{k=1}^{l^{\prime}}$ and $\left\{\mu_{k}\right\}_{k=l^{\prime}+1}^{l}$ be holomorphic frames for $\mathbb{V}_{1}$ and $\left.\operatorname{Im} \nabla v\right|_{Y}$ on $U \cap Y$, with

$$
\nabla_{\partial / \partial z_{k}(0)}^{\mathbb{V}} v=\mu_{k}(0) \quad \text { for } l^{\prime}+1 \leq k \leq l,
$$


and for $z^{\prime}=\left(z_{1}, \ldots, z_{m}\right), z^{\prime \prime}=\left(z_{m+1}, \ldots, z_{n}\right), z=\left(z^{\prime}, z^{\prime \prime}\right)$, define $\mu_{k}(z)$ by parallel transport of $\mu_{k}\left(z^{\prime}, 0\right)$ with respect to $\nabla^{\mathbb{V}}$ along the curve $u \mapsto\left(z^{\prime}, u z^{\prime \prime}\right)$. Identify $\mathbb{V}_{z}$ with $\mathbb{V}_{\left(z^{\prime}, 0\right)}$ by identifying $\mu_{k}(z)$ with $\mu_{k}\left(z^{\prime}, 0\right)$. Denote by $W_{y}(\varepsilon)$ the $\varepsilon$-neighborhood of $y$ in the normal space $N$. Then

$$
\begin{aligned}
\int_{Y \cap U} \int_{W_{y}(\varepsilon)} e^{-\frac{1}{2 t}\left(\bar{\partial}^{X} S+|v|^{2}\right)} \alpha & \\
& =\int_{Y \cap U} \int_{z \in W_{y}(\varepsilon / \sqrt{t})} e^{-\frac{1}{2 t}\left(|v(\sqrt{t} z)|^{2}+\left(\bar{\partial}^{X} S\right)(\sqrt{t} z)\right)} t^{n-m} \alpha(y, \sqrt{t} z) .
\end{aligned}
$$

Define $z=\sum_{j} z_{j}\left(\partial / \partial z_{j}\right)$ and $\bar{z}=\sum_{j} \bar{z}_{j}\left(\partial / \partial \bar{z}_{j}\right)$. The tautological vector field is $Z=z+\bar{z}$. Then, for $z \in N_{y}$,

$$
\frac{1}{2 t}|v(\sqrt{t} z)|^{2}=\frac{1}{2}\left|\nabla_{z}^{\mathbb{V}} v\right|^{2}+O(\sqrt{t})=\frac{1}{2}|z|^{2}+O(\sqrt{t})
$$

and

$$
\bar{\partial}^{X} S=\sum_{k=1}^{l}\left\langle\mu_{k}, \nabla^{\vee} v\right\rangle \mu^{k} .
$$

From now on, set $z=\left(0, z^{\prime \prime}\right)$ and $Z=z+\bar{z}$. Since $\nabla_{Z}^{\vee} \mu_{k}(0)=0$, we know that (1-6) $\frac{1}{2 t} \bar{\partial}^{X} S(\sqrt{t} z)$

$$
\begin{aligned}
& =\frac{1}{2 t} \sum_{k=1}^{l}\left\langle\mu_{k}, \nabla^{\mathbb{V}} v\right\rangle(\sqrt{t} z) \mu^{k}(0) \\
& =\frac{1}{2 t} \sum_{k=1}^{l}\left(\left\langle\mu_{k}, \nabla^{\mathbb{V}} v\right\rangle(0)+\sqrt{t}\left\langle\mu_{k}, \nabla_{Z}^{\mathbb{V}} \nabla^{\mathbb{V}} v\right\rangle(0)\right. \\
& \left.\quad+\frac{t}{2}\left(\left\langle\nabla_{Z}^{\mathbb{V}} \nabla_{Z}^{\mathbb{V}} \mu_{k}, \nabla^{\mathbb{V}} v\right\rangle+\left\langle\mu_{k}, \nabla_{Z}^{\mathbb{V}} \nabla_{Z}^{\mathbb{V}} \nabla^{\mathbb{V}} v\right\rangle\right)(0)+O\left(t^{3 / 2}\right)\right) \mu^{k}(0) .
\end{aligned}
$$

Because of the factor $t^{n-m}$ in (1-5), it should be clear that in the limit, only those monomials in the vertical form

$$
d \bar{z}_{m+1} \wedge \cdots \wedge d \bar{z}_{n} \widehat{\otimes} \mu^{l^{\prime}+1} \wedge \cdots \wedge \mu^{l}
$$

whose weight is exactly $t^{m-n}$ should be kept. Now,

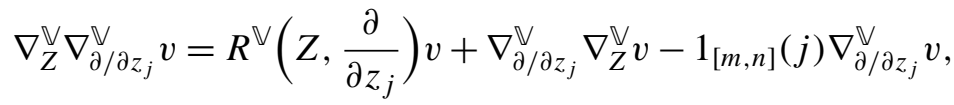

$$
\begin{aligned}
& \nabla_{\bar{z}}^{\mathbb{V}} \nabla_{\partial / \partial z_{j}}^{\mathbb{V}} v(0)=R^{\mathbb{V}}\left(\bar{z}, \frac{\partial}{\partial z_{j}}\right) v+\nabla_{\partial / \partial z_{j}}^{\mathbb{V}} \nabla_{\bar{z}}^{\mathbb{V}} v=0,
\end{aligned}
$$


where $1_{[m, n]}$ is the characteristic function of the interval $[m, n]$. Note that $\nabla^{\mathbb{V}}=$ $\nabla^{\mathbb{V}_{1}} \oplus \nabla^{\operatorname{Im} \nabla v}$ on $Y$ and that

$$
\left\langle\mu_{k}, \nabla_{z}^{\mathbb{V}} \nabla_{\partial / \partial z_{j}}^{\vee} v\right\rangle(0)=0 \quad \text { for } 1 \leq j \leq m, 1 \leq k \leq l^{\prime} .
$$

It follows that in the expression

$$
\frac{1}{2 \sqrt{t}}\left\langle\mu_{k}, \nabla_{Z}^{\mathbb{V}} \nabla^{\mathbb{V}} v\right\rangle(0) \mu^{k}(0)
$$

a nonzero contribution can only appear in the term

$$
\frac{1}{2 \sqrt{t}}\left(\sum_{j=1}^{m} \sum_{k=l^{\prime}+1}^{l}+\sum_{j=m+1}^{n} \sum_{k=1}^{l^{\prime}}\right)\left\langle\mu_{k}, \nabla_{z}^{\vee} \nabla_{\partial / \partial z_{j}}^{\mathbb{v}} v\right\rangle(0) d \bar{z}^{j} \otimes \mu^{k}(0) .
$$

Similarly, in the last term of (1-6), the only term with a nonzero contribution is

$$
\frac{1}{4} \sum_{j=1}^{m} \sum_{k=1}^{l^{\prime}}\left(\left\langle\nabla_{Z}^{\mathbb{V}} \nabla_{Z}^{\mathbb{V}} \mu_{k}, \nabla_{\partial / \partial z_{j}}^{\mathbb{V}} v\right\rangle(0)+\left\langle\mu_{k}, \nabla_{Z}^{\mathbb{V}} \nabla_{Z}^{\mathbb{V}} \nabla_{\partial / \partial z_{j}}^{\mathbb{V}} v\right\rangle(0)\right) d \bar{z}^{j} \otimes \mu^{k}(0) .
$$

But for $1 \leq j \leq m$, both $\nabla_{\partial / \partial z_{j}}^{\mathbb{V}} v(0)$ and $\nabla_{\partial / \partial z_{j}}^{\mathbb{V}} \nabla_{\bar{z}}^{\mathbb{V}} \nabla_{z}^{\mathbb{V}} v(0)=\nabla_{\partial / \partial z_{j}}^{\mathbb{V}}\left(R^{\mathbb{V}}(\bar{z}, z) v\right)(0)$ vanish, since $v=0$ on $Y$. Thus, for $1 \leq j \leq m$,

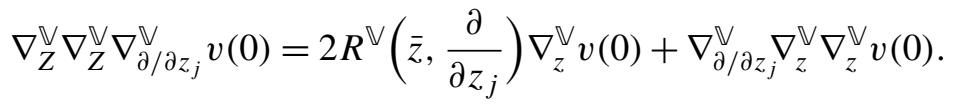

By the preceding discussion, as $t \rightarrow 0$, in (1-5), we should replace $\frac{1}{2 t} \bar{\partial}^{X} S(y, \sqrt{t} z)$ by the 2 -form

$$
\begin{aligned}
& \frac{1}{2} \sum_{k=1}^{l}\left\langle\mu_{k}, \nabla . v\right\rangle(0) \mu^{k}(0)+\sqrt{t} \times \text { expression }(1-7) \\
& +\frac{1}{2} \sum_{j=1}^{m} \sum_{k=1}^{l^{\prime}}\left\langle\mu_{k}, R^{\mathbb{V}}\left(\bar{z}, \frac{\partial}{\partial z_{j}}\right) \nabla_{z}^{\vee} v+\nabla_{\partial / \partial z_{j}}^{\mathbb{V}} \nabla_{z}^{\mathbb{V}} \nabla_{z}^{\mathbb{V}} v\right\rangle(0) d \bar{z}^{j} \otimes \mu^{k}(0) .
\end{aligned}
$$

Set $\beta_{Y}=d \bar{z}_{1} \cdots d \bar{z}_{m} \wedge \mu^{1}(0) \cdots \mu^{l^{\prime}}(0), \beta_{N}=d \bar{z}_{m+1} \cdots d \bar{z}_{n} \wedge \mu^{l^{\prime}+1}(0) \cdots \mu^{l}(0)$, $\phi\left(\mu^{1}(0) \cdots \mu^{l}(0)\right)=f d z_{1} \cdots d z_{n}$. Then

$$
\phi_{Y}\left(\mu^{1}(0) \cdots \mu^{l^{\prime}}(0)\right) \phi_{N}\left(\mu^{l^{\prime}+1}(0) \cdots \mu^{l}(0)\right)=f d z_{1} \cdots d z_{n} .
$$

Thus

$$
\begin{aligned}
\phi\left(\beta_{Y} \wedge \beta_{N}\right) & =(-1)^{l^{\prime}(n-m)} f d \bar{z}_{1} \cdots d \bar{z}_{n} \wedge d z_{1} \cdots d z_{n} \\
& =(-1)^{\left(l^{\prime}-m\right)(n-m)} \phi_{Y}\left(\beta_{Y}\right) \phi_{N}\left(\beta_{N}\right) .
\end{aligned}
$$

Now, observing that $\int_{\mathbb{C}} \bar{z}^{i} e^{-|z|^{2}} d z d \bar{z}=0$ for $i>0$ and that $\nabla . v v:\left(N, g_{1}^{N}\right) \rightarrow$ $\left(\operatorname{Im} \nabla v, h^{\operatorname{Im} \nabla v}\right)$ is an isometry and $l-l^{\prime}=n-m$, we find that the limit of (1-4) 
as $t \rightarrow 0$ is the sum over $j$ of

$$
\begin{aligned}
& \text { (1-8) } \int_{Y_{j}}(-1)^{(l-n)(n-m)} j^{*} \alpha \int_{N} \exp \left(-\frac{1}{2} \sum_{k=1}^{l}\left\langle\mu_{k}, \nabla^{\mathbb{V}} v\right\rangle(0) \mu^{k}(0)\right. \\
& \left.-\frac{1}{2}\left\langle\cdot, P^{\mathbb{V}_{1}} R^{\mathbb{V}}\left(\bar{z}, j_{*} \cdot\right) \nabla_{z}^{\mathbb{V}} v\right\rangle(0)-\frac{1}{2}\left|\nabla_{z}^{\mathbb{V}} v\right|^{2}\right) .
\end{aligned}
$$

The second integrand in this expression can be rewritten as

$$
\begin{aligned}
& \exp \left(-\frac{1}{2} \sum_{i=1}^{n-m} d \bar{z}_{m+i} \wedge \mu^{l^{\prime}+i}(0)+\frac{1}{2}\left\langle R^{\mathbb{V}}\left(z, j_{*} \cdot\right) P^{\mathbb{V}_{1}} \cdot, \nabla_{z}^{\mathbb{V}} v\right\rangle(0)-\frac{1}{2}|z|^{2}\right) \\
& \quad=\exp \left(\frac{1}{2}\left\langle\left(\nabla^{\mathbb{V}} v\right)^{-1} R^{\mathbb{V}}\left(z, j_{*} \cdot\right) P^{\mathbb{V}_{1}} \cdot, z\right\rangle-\frac{1}{2}|z|^{2}\right)\left(\frac{1}{2}\right)^{l-l^{\prime}} d z_{m+1} d \bar{z}_{m+1} \cdots d z_{n} d \bar{z}_{n} .
\end{aligned}
$$

Thus the expression in (1-8) is equal to

$$
\int_{Y_{j}} \frac{(-1)^{(l-n)(n-m)} \alpha}{\operatorname{det}_{N}\left(\left(1+R_{v}^{\mathbb{V}}\right) /(-2 \pi i)\right)},
$$

which leads to (1-3).

\section{Localization of Quillen metrics via a transversal section}

Let $X$ be a compact complex manifold of dimension $n$. Let $\mathbb{V}$ and $\xi$ be holomorphic vector bundles on $X$ with $\operatorname{dim} \mathbb{V}=m$, and let $v$ be a holomorphic section of $\mathbb{V}$. Assume that $v$ vanishes on a complex manifold $Y \subset X$ and satisfies (1-1). Then we have a complex of holomorphic vector bundles on $X$,

$$
0 \rightarrow \bigwedge^{m}\left(\mathbb{V}^{*}\right) \stackrel{i(v)}{\longrightarrow} \bigwedge^{m-1}\left(\mathbb{V}^{*}\right) \stackrel{i(v)}{\longrightarrow} \cdots \stackrel{i(v)}{\longrightarrow} \bigwedge^{1}\left(\mathbb{V}^{*}\right) \stackrel{i(v)}{\longrightarrow} \bigwedge^{0}\left(\mathbb{V}^{*}\right) \rightarrow 0 .
$$

Let $\left(\Omega\left(X, \wedge\left(\mathbb{V}^{*}\right) \otimes \xi\right), \bar{\partial}^{X}\right)$ be the Dolbeault complex associated to the holomorphic vector bundle $\wedge\left(\mathbb{V}^{*}\right) \otimes \xi$. Let $\mathscr{H}_{v}\left(X, \wedge\left(\mathbb{V}^{*}\right) \otimes \xi\right)$ be the hypercohomologies of the bicomplex $\left(\Omega\left(X, \wedge\left(\mathbb{V}^{*}\right) \otimes \xi\right), \bar{\partial}^{X}, i(v)\right)$. Let $j: Y \rightarrow X$ be the obvious embedding. Now the pullback map $j^{*}$ induces naturally a map of complexes

$$
j^{*}:\left(\Omega\left(X, \wedge\left(\mathbb{V}^{*}\right) \otimes \xi\right), \bar{\partial}^{X}+i(v)\right) \rightarrow\left(\Omega\left(Y, \wedge\left(\mathbb{V}_{1}^{*}\right) \otimes \xi\right), \bar{\partial}^{Y}\right) .
$$

Theorem 2.1. The map $j^{*}$ is a quasi-isomorphism of complexes. In particular, $j^{*}$ induces an isomorphism

$$
\mathscr{H}_{v}\left(X, \wedge\left(\mathbb{V}^{*}\right) \otimes \xi\right) \simeq H\left(Y, \wedge\left(\mathbb{V}_{1}^{*}\right) \otimes \xi\right)
$$

Proof. In [Feng 2003] there is an analytic proof of this theorem when $\mathbb{V}=T X$. There we used the twisted vector bundle $\wedge\left(T^{*} X\right)$ and here $\wedge\left(\mathbb{V}^{*}\right)$ takes its place; the proof works just the same. For an algebraic proof, we can modify the proof of [Bismut 2004, Theorem 5.1]. 
Let $N^{X}, N_{H}^{X}$ be the number operators on $\wedge\left(T^{*} X\right), \wedge\left(\mathbb{V}^{*}\right)$ corresponding to multiplication by $p$ on $\wedge^{p}\left(T^{*} X\right), \wedge^{p}\left(\mathbb{V}^{*}\right)$; do the same replacing $X$ by $Y$ and $\mathbb{V}^{*}$ by $\mathbb{V}_{1}^{*}$. Then $N^{X}-N_{H}^{X}$ and $N^{Y}-N_{H}^{Y}$ define $\mathbb{Z}$-gradings on $\Omega\left(X, \wedge\left(\mathbb{V}^{*}\right) \otimes \xi\right)$ and $\Omega\left(Y, \wedge\left(\mathbb{V}_{1}^{*}\right) \otimes \xi\right)$, which in turn induce $\mathbb{Z}$-gradings on $\mathscr{H}_{v}\left(X, \wedge\left(\mathbb{V}^{*}\right) \otimes \xi\right)$ and $H\left(Y, \wedge\left(\mathbb{V}_{1}^{*}\right) \otimes \xi\right)$, respectively. The isomorphism $j^{*}$ preserves these $\mathbb{Z}$-gradings.

From [Bismut and Lebeau 1991, (1.24)], we define the complex lines $\lambda_{v}\left(\mathbb{V}^{*}\right)$ and $\lambda\left(\mathbb{V}_{1}^{*}\right)$ by

$$
\begin{aligned}
& \lambda_{v}\left(\mathbb{V}^{*}\right)=\bigotimes_{p=-m}^{n}\left(\operatorname{det} \mathscr{H}_{v}^{p}\left(X, \wedge\left(\mathbb{V}^{*}\right) \otimes \xi\right)\right)^{(-1)^{p+1}}, \\
& \lambda\left(\mathbb{V}_{1}^{*}\right)=\bigotimes_{p=0}^{n} \bigotimes_{q=0}^{m}\left(\operatorname{det} H^{p}\left(Y, \wedge^{q}\left(\mathbb{V}_{1}^{*}\right) \otimes \xi\right)\right)^{(-1)^{p+q+1}} .
\end{aligned}
$$

By (2-3), we have a canonical isomorphism of complex lines

$$
\lambda_{v}\left(\mathbb{V}^{*}\right) \simeq \lambda\left(\mathbb{V}_{1}^{*}\right)
$$

Let $\rho$ be the nonzero section of $\lambda\left(\mathbb{V}_{1}^{*}\right)^{-1} \otimes \lambda_{v}\left(\mathbb{V}^{*}\right)$ associated with this canonical isomorphism.

Let $g^{T X}$ be a Kähler metric on $T X$. We identify $N$ with the bundle orthogonal to $T Y$ in $\left.T X\right|_{Y}$. Let $g^{T Y}$ and $g^{N}$ be the metrics on $T Y$ and $N$ induced by $g^{T X}$. Let $h^{\xi}$ be a Hermitian metric on $\xi$. Let $h^{\mathbb{V}}$ be a metric on $\mathbb{V}$ such that $\mathbb{V}_{1}$ and $\left.\operatorname{Im} \nabla v\right|_{Y}$ are orthogonal on $Y$ and $\left.\nabla v\right|_{Y}:\left.N \rightarrow \operatorname{Im} \nabla v\right|_{Y}$ is an isometry.

Let $d v_{X}$ be the Riemannian volume form on $\left(X, g^{T X}\right)$. Let $\langle\cdot, \cdot\rangle_{0}$ be the metric on $\wedge\left(\overline{T^{*} X}\right) \widehat{\otimes} \wedge\left(\mathbb{V}^{*}\right) \otimes \xi$ induced by $g^{T X}, h^{\mathbb{V}}, h^{\xi}$. The Hermitian product on $\Omega\left(X, \wedge\left(\mathbb{V}^{*}\right) \otimes \xi\right)$ is defined by

$$
\left\langle\alpha, \alpha^{\prime}\right\rangle=\frac{1}{(2 \pi)^{n}} \int_{X}\left\langle\alpha, \alpha^{\prime}\right\rangle_{0} d v_{X} \quad \text { for } \alpha, \alpha^{\prime} \in \Omega\left(X, \wedge\left(\mathbb{V}^{*}\right) \otimes \xi\right) .
$$

Let $\bar{\partial}^{X *}$ and $v^{*} \wedge=i(v)^{*}$ be the adjoint of $\bar{\partial}^{X}$ and $i(v)$ with respect to $\langle\cdot, \cdot\rangle$. Set

$$
V=i(v)+i(v)^{*}, \quad D^{X}=\bar{\partial}^{X}+\bar{\partial}^{X *} .
$$

By Hodge theory,

$$
\mathscr{H}_{v}\left(X, \wedge\left(\mathbb{V}^{*}\right) \otimes \xi\right) \simeq \operatorname{Ker}\left(D^{X}+V\right) .
$$

Denote by $P$ be the operator of orthogonal projection from $\Omega\left(X, \wedge\left(\mathbb{V}^{*}\right) \otimes \xi\right)$ onto $\operatorname{ker}\left(D^{X}+V\right)$ and set $P^{\perp}=1-P$. Let $h^{\mathscr{H}_{v}}$ be the $L^{2}$-metric on $\mathscr{H}_{v}\left(X, \wedge\left(\mathbb{V}^{*}\right) \otimes\right.$ $\xi$ ) induced by the $L^{2}$-product (2-4) via the isomorphism (2-5). Define in the same way a Hermitian product on $\Omega\left(Y, \wedge\left(\mathbb{V}_{1}^{*}\right) \otimes \xi\right.$ ) associated to $g^{T Y}, h^{\mathbb{V}_{1}}, h^{\xi}$. Let $\bar{\partial}^{Y *}$ be the adjoint of $\bar{\partial}^{Y}$, and $h^{H\left(Y, \wedge\left(\mathbb{V}_{1}^{*}\right) \otimes \xi\right)}$ the corresponding $L^{2}$-metric on 
$H\left(Y, \wedge\left(\mathbb{V}_{1}^{*}\right) \otimes \xi\right)$. Set

$$
D^{Y}=\bar{\partial}^{Y}+\bar{\partial}^{Y *} .
$$

Let $Q$ be the orthogonal projection operator from $\Omega\left(Y, \wedge\left(\mathbb{V}_{1}^{*}\right) \otimes \xi\right)$ on $\operatorname{Ker} D^{Y}$, and $Q^{\perp}=1-Q$. Let $|\cdot|_{\lambda_{v}\left(\mathbb{V}^{*}\right)}$ and $|\cdot|_{\lambda\left(\mathbb{V}^{*}\right)}$ be the $L^{2}$-metrics on $\lambda_{v}\left(\mathbb{V}^{*}\right)$ and $\lambda\left(\mathbb{V}^{*}\right)$ induced by $h^{\mathscr{H} \mathcal{L}_{v}}$ and $h^{H\left(Y, \wedge\left(\mathbb{V}_{1}^{*}\right) \otimes \xi\right)}$. Following [Bismut and Lebeau 1991, (1.49)], let

$$
\theta_{v}^{X}(s)=-\operatorname{Tr}_{s}\left(\left(N^{X}-N_{H}^{X}\right)\left(\left(D^{X}+V\right)^{2}\right)^{-s} P^{\perp}\right) .
$$

Then $\theta_{v}^{X}(s)$ extends to a meromorphic function of $s \in \mathbb{C}$, which is holomorphic at $s=0$.

The Quillen metric $\|\cdot\|_{\lambda_{v}}\left(\mathbb{V}^{*}\right)$ on the line $\lambda_{v}\left(\mathbb{V}^{*}\right)$ is defined by

$$
\|\cdot\|_{\lambda_{v}\left(\mathbb{V}^{*}\right)}=|\cdot|_{\lambda_{v}\left(\mathbb{V}^{*}\right)} \exp \left(-\frac{1}{2} \frac{\partial \theta_{v}^{X}}{\partial s}(0)\right) .
$$

In the same way, the function

$$
\theta^{Y}(s)=-\operatorname{Tr}_{s}\left(\left(N^{Y}-N_{H}^{Y}\right)\left(D^{Y, 2}\right)^{-s} Q^{\perp}\right)
$$

extends to a meromorphic function of $s \in \mathbb{C}$, holomorphic at $s=0$. The Quillen metric $\|\cdot\|_{\lambda\left(\mathbb{V}_{1}^{*}\right)}$ on the line $\lambda\left(\mathbb{V}_{1}^{*}\right)$ is defined by

$$
\|\cdot\|_{\lambda\left(\mathbb{V}_{1}^{*}\right)}=|\cdot|_{\lambda\left(\mathbb{V}_{1}^{*}\right)} \exp \left(-\frac{1}{2} \frac{\partial \theta^{Y}}{\partial s}(0)\right) .
$$

Let $\|\cdot\|_{\lambda\left(\mathbb{V}_{1}^{*}\right)^{-1} \otimes \lambda_{v}\left(\mathbb{V}^{*}\right)}$ be the Quillen metric on $\lambda\left(\mathbb{V}_{1}^{*}\right)^{-1} \otimes \lambda_{v}\left(\mathbb{V}^{*}\right)$ induced by $\|\cdot\|_{\lambda_{v}\left(\mathbb{V}^{*}\right)}$ and $\|\cdot\|_{\lambda\left(\mathbb{V}_{1}^{*}\right)}$ as in [Bismut and Lebeau 1991, §1e].

The purpose of this section is to give a formula for $\|\rho\|_{\lambda\left(\mathbb{V}_{1}^{*}\right)^{-1} \otimes \lambda_{v}\left(\mathbb{V}^{*}\right)}^{2}$. Now we introduce some notations.

For a holomorphic Hermitian vector bundle $\left(E, h^{E}\right)$ on $X$, we denote by $\operatorname{Td}(E)$, $\operatorname{ch}(E), c_{\max }(E)$ the Todd class, Chern character, and top Chern class of $E$, and by $\operatorname{Td}\left(E, h^{E}\right), \operatorname{ch}\left(E, h^{E}\right), c_{\max }\left(E, h^{E}\right)$ the Chern-Weil representatives of $\operatorname{Td}(E)$, $\operatorname{ch}(E), c_{\max }(E)$ with respect to the holomorphic Hermitian connection $\nabla^{E}$ on $\left(E, h^{E}\right)$.

Let $\delta_{Y}$ be the current of integration on $Y$. By [Bismut 1992, Theorem 3.6], a current $\tilde{c}_{\max }\left(\mathbb{V}, h^{\mathbb{V}}\right)$ on $X$ is well defined by the holomorphic section $v$ (which induces an embedding $v: X \rightarrow \mathbb{V}$ ), and this current satisfies

$$
\frac{\bar{\partial} \partial}{2 \pi i} \tilde{c}_{\max }\left(\mathbb{V}, h^{\mathbb{V}}\right)=c_{\max }\left(\mathbb{V}_{1}, h^{\mathbb{V}_{1}}\right) \delta_{Y}-c_{\max }\left(\mathbb{V}, h^{\mathbb{V}}\right) .
$$

Let $\widetilde{T d}\left(T Y, T X, g^{\left.T X\right|_{Y}}\right)$ be the Bott-Chern current on $Y$ associated to the exact sequence

$$
\left.0 \rightarrow T Y \rightarrow T X\right|_{Y} \rightarrow N \rightarrow 0
$$


constructed in [Bismut et al. 1988a, §1f], which satisfies

$$
\frac{\bar{\partial} \partial}{2 \pi i} \widetilde{\mathrm{Td}}\left(T Y, T X, g^{\left.T X\right|_{Y}}\right)=\operatorname{Td}\left(\left.T X\right|_{Y}, g^{\left.T X\right|_{Y}}\right)-\operatorname{Td}\left(T Y, g^{T Y}\right) \operatorname{Td}\left(N, g^{N}\right) .
$$

Finally, let $R(x)$ be the power series introduced in [Gillet and Soule 1991], which is such that if $\zeta(s)$ is the Riemann zeta function, then

$$
R(x)=\sum_{\substack{n \geq 1 \\ n \text { odd }}}\left(\sum_{j=1}^{n} \frac{1}{j} \zeta(-n)+2 \frac{\partial \zeta}{\partial s}(-n)\right) \frac{x^{n}}{n !} .
$$

We identify $R$ with the corresponding additive genus. We also set

$$
\operatorname{ch}\left(\bigwedge^{*}\left(\mathbb{V}_{1}^{*}\right)\right)=\sum_{i}(-1)^{i} \operatorname{ch}\left(\bigwedge^{i}\left(\mathbb{V}_{1}^{*}\right)\right),
$$

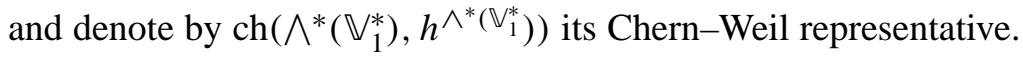

Theorem 2.2. The Quillen metric $\|\rho\|_{\lambda\left(\mathbb{V}_{1}^{*}\right)^{-1} \otimes \lambda_{v}\left(\mathbb{V}^{*}\right)}^{2}$ is given by the exponential of

$$
\begin{aligned}
& -\int_{X} \operatorname{Td}\left(T X, g^{T X}\right) \operatorname{Td}^{-1}\left(\mathbb{V}, h^{\mathbb{V}}\right) \tilde{c}_{\max }\left(\mathbb{V}, h^{\mathbb{V}}\right) \operatorname{ch}\left(\xi, h^{\xi}\right) \\
& +\int_{Y} \operatorname{Td}^{-1}\left(N, g^{N}\right) \widetilde{\operatorname{Td}}\left(T Y,\left.T X\right|_{Y}, g^{\left.T X\right|_{Y}}\right) \operatorname{ch}\left(\wedge^{*}\left(\mathbb{V}_{1}^{*}\right), h^{\wedge^{*}\left(\mathbb{V}_{1}^{*}\right)}\right) \operatorname{ch}\left(\xi, h^{\xi}\right) \\
& -\int_{Y} \operatorname{Td}(T Y) R(N) \operatorname{ch}\left(\wedge^{*}\left(\mathbb{V}_{1}^{*}\right)\right) \operatorname{ch}(\xi) .
\end{aligned}
$$

Proof. Set

$$
T\left(\bigwedge\left(\mathbb{V}^{*}\right), h^{\wedge\left(\mathbb{V}^{*}\right)}\right)=\operatorname{Td}^{-1}\left(\mathbb{V}, h^{\mathbb{V}}\right) \tilde{c}_{\max }\left(\mathbb{V}, h^{\mathbb{V}}\right) .
$$

By the same argument as in [Bismut et al. 1990, Theorem 3.17], the current

$$
T\left(\wedge\left(\mathbb{V}^{*}\right), h^{\wedge\left(\mathbb{V}^{*}\right)}\right)
$$

is exactly the current on $X$ associated to (2-1) (evaluated modulo irrelevant $\partial$ or $\bar{\partial}$ coboundaries).

Now, from the choice of our metric $h^{\mathbb{V}}$, the analogue of [Bismut and Lebeau 1991, Definition 1.21, assumption (A)] is satisfied for the complex (2-1). Then we verify that as far as local index theoretic computations are concerned, the situation is exactly the same as in [Bismut and Lebeau 1991]. Because of the quasi-isomorphism of Theorem 2.1, there are no "small" eigenvalues of the operator $D+T V$ when $T \rightarrow+\infty$. In Section 3, we write down the intermediate results corresponding to [Bismut and Lebeau 1991, §6c]. Comparing to [Bismut and Lebeau 1991, §§6c-6e], the proof of Theorem 2.2 is complete. 
Remark 2.3. Assume that $Y$ consists only discrete points; then $l \geq n$ and the last two terms of (2-8) are zero. In this case, if $n=l$, then (2-1) is a resolution of $j_{*}\left(O_{Y}\right)$ and Theorem 2.2 is a direct consequence of [Bismut and Lebeau 1991,

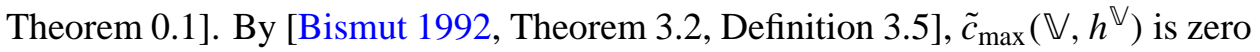
if $l>n+1$.

\section{3. $L^{2}$ metrics on $H_{v}\left(X, \wedge\left(\mathbb{V}^{*}\right)\right)$ and localization}

We keep the assumptions and notations of Section 2.

Let $g^{T X}$ be a Kähler metric on $T X$, and let $g^{T Y}, g^{N}$ be the metrics on $T Y, N$ induced by $g^{T X}$. Let $h^{\mathbb{V}}$ be a metric on $\mathbb{V}$ such that $\mathbb{V}_{1}$ and $\left.\operatorname{Im} \nabla v\right|_{Y}$ are orthogonal on $Y$ and $\left.\nabla v\right|_{Y}:\left.\left(N, g^{N}\right) \rightarrow \operatorname{Im} \nabla v\right|_{Y}$ is an isometry.

Let $\phi_{1}: \operatorname{det} \mathbb{V}_{1}^{*} \rightarrow \operatorname{det} T^{*} Y$ be a nonzero holomorphic section. Let $h_{1}^{\mathbb{V}}$ be a metric on $\mathbb{V}$ such that on $Y, \mathbb{V}_{1}$ and $\left.\operatorname{Im} \nabla v\right|_{Y}$ are orthogonal and

$$
|\phi|_{\operatorname{det} \mathbb{V} \otimes \operatorname{det} T^{*} X, 1}=\left|\phi_{1}\right|_{\operatorname{det} \mathbb{V}} \otimes \operatorname{det} T^{*} Y, 1=1,
$$

where $|\cdot|_{\operatorname{det} \mathbb{V} \otimes \operatorname{det} T^{*} X, 1}$ and $|\cdot|_{\operatorname{det} \mathbb{V}_{1} \otimes \operatorname{det} T^{*} Y, 1}$ are the norms on the holomorphic line bundles det $\mathbb{V} \otimes \operatorname{det} T^{*} X$ and $\operatorname{det} \mathbb{V}_{1} \otimes \operatorname{det} T^{*} Y$ induced by $h_{1}^{\vee}$ and $g^{T X}$.

We will add a subscript 1 to denote the objects induced by $h_{1}^{\vee}$. For

$$
\beta \in \bigwedge^{p}\left(\overline{T^{*} X}\right) \widehat{\otimes} \bigwedge^{q}\left(\mathbb{V}^{*}\right),
$$

we define $* \mathbb{V}, 1 \beta \in \bigwedge^{n-p}\left(\overline{T^{*} X}\right) \widehat{\otimes} \wedge^{l-q}\left(\mathbb{V}^{*}\right)$ by

$$
\langle\alpha, \beta\rangle_{1} \phi^{-1}\left(d v_{X}\right)=\alpha \wedge * \mathbb{V}, 1 \beta .
$$

It's useful to write down a local expression for $* \mathbb{V}, 1$. if $\left\{w^{i}\right\}_{i=1}^{n}$ and $\left\{\mu^{i}\right\}_{i=1}^{l}$, are

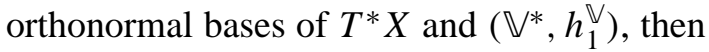

$$
d v_{X}=(-1)^{n(n+1) / 2}(\sqrt{-1})^{n} \bar{w}^{1} \wedge \cdots \wedge \bar{w}^{n} \widehat{\otimes} w^{1} \wedge \cdots \wedge w^{n}
$$

and $\phi^{-1}\left(w^{1} \wedge \cdots \wedge w^{n}\right)=f \mu^{1} \wedge \cdots \wedge \mu^{l}$ with $|f|=1$. If

$$
\beta=\bar{w}^{1} \wedge \cdots \wedge \bar{w}^{p} \widehat{\otimes} \mu^{1} \wedge \cdots \wedge \mu^{q},
$$

then

$$
* \mathbb{v}, 1 \beta=(-1)^{(n-p) q+n(n+1) / 2}(\sqrt{-1})^{n} f \bar{w}^{p+1} \wedge \cdots \wedge \bar{w}^{n} \widehat{\otimes} \mu^{q+1} \wedge \cdots \wedge \mu^{l} .
$$

Thus $* \mathbb{V}, 1 * \mathbb{V}, 1 \beta=(-1)^{(p+q)(n+l+1)} \beta$, for any $\beta \in \bigwedge^{p}\left(\overline{T^{*} X}\right) \widehat{\otimes} \wedge^{q}\left(\mathbb{V}^{*}\right)$. Combining this with (1-2), we find that

$$
\bar{\partial}^{X *} \beta=(-1)^{p+q+1} *_{\mathbb{V}, 1}^{-1} \bar{\partial}^{X} * \mathbb{V}, 1 \beta, \quad(i(v))^{*} \beta=(-1)^{p+q+1} *_{\mathbb{V}, 1}^{-1} i(v) * \mathbb{V}, 1 \beta .
$$

Thus the antilinear map $* \mathbb{V}, 1$ is an isometry from $\left(\mathscr{H}_{v}\left(X, \wedge\left(\mathbb{V}^{*}\right)\right), h_{1}^{\mathscr{H}_{v}}\right)$ to itself. 
The bilinear form

$$
\alpha, \beta \in \mathscr{H}_{v}\left(X, \wedge\left(\mathbb{V}^{*}\right)\right) \mapsto \frac{1}{(2 \pi)^{n}} \int_{X} \alpha \wedge \beta
$$

is nondegenerate; indeed, $\alpha \in \mathscr{H}_{v}\left(X, \wedge\left(\mathbb{V}^{*}\right)\right)$ implies $* \mathbb{V}, 1 \alpha \in \mathscr{H}_{v}\left(X, \wedge\left(\mathbb{V}^{*}\right)\right)$, so $\alpha \neq 0$ implies

$$
\int_{X} \alpha \wedge * \mathbb{v}, 1 \alpha>0
$$

Thus the metric $|\cdot|_{\lambda_{v}}\left(\mathbb{V}^{*}\right), 1$ on $\lambda_{v}\left(\mathbb{V}^{*}\right)$ only depends on the nondegenerate bilinear form $(3-1)$ on $\mathscr{H}_{v}\left(X, \wedge\left(\mathbb{V}^{*}\right)\right)$, which is metric-independent.

Recall the definition of det $\left.\nabla v\right|_{Y}$ from Section 1. Now,

$$
\frac{\left.\phi\right|_{Y} /\left(\left(\left.\operatorname{det} \nabla v\right|_{Y}\right)^{*}\right)}{\phi_{1}}
$$

is a holomorphic function on $Y$. Since $Y$ is compact, this function is locally constant. Then we have the following extension of [Bismut 2004, Theorem 5.7].

\section{Theorem 3.1.}

(3-2) $\log \left(|\rho|_{\lambda\left(\mathbb{V}_{1}^{*}\right)^{-1} \otimes \lambda_{v}\left(\mathbb{V}^{*}\right), 1}\right)^{2}=\int_{Y} \operatorname{Td}(T Y) \operatorname{ch}\left(\bigwedge\left(\mathbb{V}_{1}^{*}\right)\right) \log \left|\frac{\left.\phi\right|_{Y} /\left(\left(\left.\operatorname{det} \nabla v\right|_{Y}\right)^{*}\right)}{\phi_{1}}\right|$.

Proof. We use $\phi_{1}$ to define the integral $\int_{Y} \gamma$ for $\gamma \in H\left(Y, \wedge\left(\mathbb{V}_{1}^{*}\right)\right)$. Since

$$
\left|\phi_{1}\right|_{\operatorname{det} \mathbb{V}_{1} \otimes \operatorname{det} T^{*} Y, 1}=1,
$$

following the same considerations as above, we find that the antilinear operator $* \mathbb{V}_{1}, 1$ maps $H\left(Y, \wedge\left(\mathbb{V}_{1}^{*}\right)\right)$ into itself isometrically. Therefore, to evaluate the lefthand side of (3-2), we only need to compare the bilinear forms (3-1) with

$$
a, b \in H\left(Y, \wedge\left(\mathbb{V}_{1}^{*}\right)\right) \mapsto \frac{1}{(2 \pi)^{m}} \int_{Y} a \wedge b .
$$

Let $A_{v} \in \operatorname{End}^{\text {even }} H\left(Y, \wedge\left(\mathbb{V}_{1}^{*}\right)\right)$ be given by

$$
a \rightarrow \frac{(-1)^{(l-n)(n-m)} a}{(2 \pi)^{n-m} \operatorname{det}_{N}\left(\left(1+R_{v}^{\mathbb{V}}\right) /(-2 \pi i)\right)} \frac{\left.\phi\right|_{Y} /\left(\left(\left.\operatorname{det} \nabla v\right|_{Y}\right)^{*}\right)}{\phi_{1}} .
$$

Set

then

$$
\operatorname{det} A_{v}=\frac{\left.\operatorname{det} A_{v}\right|_{H^{\text {even }}\left(Y, \wedge\left(\mathbb{V}_{1}^{*}\right)\right)}}{\left.\operatorname{det} A_{v}\right|_{H^{\text {odd }}\left(Y, \wedge\left(\mathbb{V}_{1}^{*}\right)\right)}} ;
$$

$$
\left(|\rho|_{\lambda\left(\mathbb{V}_{1}^{*}\right)^{-1} \otimes \lambda_{v}\left(\mathbb{V}^{*}\right), 1}\right)^{2}=\left|\operatorname{det} A_{v}\right| .
$$

Now, $A_{v}$ is a degree-increasing operator in $H\left(Y, \wedge\left(\mathbb{V}_{1}^{*}\right)\right)$. Therefore it acts like a triangular matrix whose diagonal part is just multiplication by the locally constant 
function $\frac{\left.\phi\right|_{Y} /\left(\left(\left.\operatorname{det} \nabla v\right|_{Y}\right)^{*}\right)}{\phi_{1}}$. Using (3-3), we get

$$
\operatorname{det} A_{v}=\left(\frac{\left.\phi\right|_{Y} /\left(\left(\left.\operatorname{det} \nabla v\right|_{Y}\right)^{*}\right)}{\phi_{1}}\right)^{\chi\left(Y, \wedge\left(\mathbb{V}_{1}^{*}\right)\right)} .
$$

But $\chi\left(Y, \wedge\left(\mathbb{V}_{1}^{*}\right)\right)=\int_{Y} \operatorname{Td}(T Y) \operatorname{ch}\left(\bigwedge\left(\mathbb{V}_{1}^{*}\right)\right)$; thus we get (3-2).

Let $g_{1}^{N}$ be the metric on $N$ such that $\left.\nabla v\right|_{Y}:\left(N, g_{1}^{N}\right) \rightarrow\left(\operatorname{Im}(\nabla v), h_{1}^{\operatorname{Im}(\nabla v)}\right)$ is an isometry. Let $\widetilde{\mathrm{T}}^{-1}\left(N, g^{N}, g_{1}^{N}\right)$ be the Bott-Chern class constructed in [Bismut et al. 1988a, §1f] such that

$$
\frac{\bar{\partial} \partial}{2 \pi i} \mathrm{~T}^{-1}\left(N, g^{N}, g_{1}^{N}\right)=\mathrm{Td}^{-1}\left(N, g_{1}^{N}\right)-\mathrm{Td}^{-1}\left(N, g^{N}\right) .
$$

Finally, we can compute the analytic torsion on the total manifold via the zero set of a transversal section $v$.

Theorem 3.2. If $h_{1}^{\mathbb{V}_{1}}=h^{\mathbb{V}_{1}}$ on $Y$, then

$$
\begin{gathered}
-\frac{\partial \theta_{v, 1}^{X}}{\partial s}(0)+\frac{\partial \theta^{Y}}{\partial s}(0)=-\int_{X} \operatorname{Td}\left(T X, g^{T X}\right) \operatorname{Td}^{-1}\left(\mathbb{V}, h_{1}^{\mathbb{V}}\right) \tilde{c}_{\max }\left(\mathbb{V}, h_{1}^{\mathbb{V}}\right) \\
+\int_{Y}\left(\operatorname{Td}^{-1}\left(N, g^{N}\right) \widetilde{\operatorname{Td}}\left(T Y,\left.T X\right|_{Y}, g^{\left.T X\right|_{Y}}\right)\right. \\
\left.+\operatorname{Td}\left(T X, g^{T X}\right) \widetilde{T d}^{-1}\left(N, g^{N}, g_{1}^{N}\right)\right) \operatorname{ch}\left(\wedge^{*}\left(\mathbb{V}_{1}^{*}\right), h^{\wedge^{*}\left(\mathbb{V}_{1}^{*}\right)}\right) \\
-\int_{Y} \operatorname{Td}(T Y) \operatorname{ch}\left(\wedge^{*}\left(\mathbb{V}_{1}^{*}\right)\right)\left(R(N)+\log \left|\frac{\left.\phi\right|_{Y} /\left(\left(\left.\operatorname{det} \nabla v\right|_{Y}\right)^{*}\right)}{\phi_{1}}\right|\right) .
\end{gathered}
$$

Proof. Since $h_{1}^{\mathbb{V}_{1}}=h^{\mathbb{V}_{1}}$, we have $|\cdot|_{\lambda\left(\mathbb{V}_{1}^{*}\right)}=|\cdot|_{\lambda\left(\mathbb{V}_{1}^{*}\right), 1}$ and $\|\cdot\|_{\lambda\left(\mathbb{V}_{1}^{*}\right)}=\|\cdot\|_{\lambda\left(\mathbb{V}_{1}^{*}\right), 1}$. Let $\tilde{\mathrm{ch}}\left(\wedge\left(\mathbb{V}^{*}\right), h_{1}^{\wedge\left(\mathbb{V}^{*}\right)}, h^{\wedge\left(\mathbb{V}^{*}\right)}\right)$ be the Bott-Chern class constructed in [Bismut et al. 1988a, §1f], so that

$$
\frac{\bar{\partial} \partial}{2 \pi i} \tilde{\operatorname{ch}}\left(\wedge\left(\mathbb{V}^{*}\right), h_{1}^{\wedge\left(\mathbb{V}^{*}\right)}, h^{\wedge\left(\mathbb{V}^{*}\right)}\right)=\operatorname{ch}\left(\wedge\left(\mathbb{V}^{*}\right), h^{\wedge\left(\mathbb{V}^{*}\right)}\right)-\operatorname{ch}\left(\wedge\left(\mathbb{V}^{*}\right), h_{1}^{\wedge\left(\mathbb{V}^{*}\right)}\right) .
$$

Then by the anomaly formula [Bismut et al. 1988b, Theorem 1.23],

$$
\log \left(\frac{\|\cdot\|_{\lambda_{v}\left(\mathbb{V}^{*}\right)}^{2}}{\|\cdot\|_{\lambda_{v}\left(\mathbb{V}^{*}\right), 1}^{2}}\right)=\int_{X} \operatorname{Td}\left(T X, g^{T X}\right) \widetilde{c h}\left(\wedge\left(\mathbb{V}^{*}\right), h_{1}^{\wedge\left(\mathbb{V}^{*}\right)}, h^{\wedge\left(\mathbb{V}^{*}\right)}\right) .
$$

By [Bismut et al. 1990, Theorem 2.5],

$$
\begin{aligned}
& T\left(\wedge\left(\mathbb{V}^{*}\right), h^{\wedge\left(\mathbb{V}^{*}\right)}\right)-T\left(\wedge\left(\mathbb{V}^{*}\right), h_{1}^{\wedge\left(\mathbb{V}^{*}\right)}\right) \\
& =\operatorname{ch}\left(\wedge^{*}\left(\mathbb{V}_{1}^{*}\right), h^{\wedge^{*}\left(\mathbb{V}_{1}^{*}\right)}\right) \mathrm{T}{\widetilde{d^{-1}}}^{-1}\left(N, g_{1}^{N}, g^{N}\right) \delta_{Y}-\widetilde{\operatorname{ch}}\left(\wedge\left(\mathbb{V}^{*}\right), h_{1}^{\wedge\left(\mathbb{V}^{*}\right)}, h^{\wedge\left(\mathbb{V}^{*}\right)}\right) .
\end{aligned}
$$

By (2-9), Theorems 2.2 and 3.1, and the preceding equations, the proof of Theorem 3.2 is complete. 
Remark 3.3. If $Y$ consists only of discrete points and $n=l$, then $\phi_{1}=\mathrm{Id}$. In this case let $g^{\operatorname{det} N}$ and $g_{1}^{\operatorname{det} N}$ be the metrics on $\operatorname{det} N=\operatorname{det} T X$ induced by $g^{N}$ and $g_{1}^{N}$. By Remark 2.3 and Theorem 3.2,

$$
\begin{aligned}
-\frac{\partial \theta_{v, 1}^{X}}{\partial s}(0)=-\int_{X} \operatorname{Td}\left(T X, g^{T X}\right) & \operatorname{Td}^{-1}\left(\mathbb{V}, h_{1}^{\mathbb{V}}\right) \tilde{c}_{\max }\left(\mathbb{V}, h_{1}^{\vee}\right) \\
& +\sum_{p \in Y}\left(\frac{1}{2} \log \left(g^{\operatorname{det} N} / g_{1}^{\operatorname{det} N}\right)-\log \left|\phi /\left(\left.\operatorname{det} \nabla v\right|_{Y}\right)^{*}\right|\right) .
\end{aligned}
$$

Remark 3.4. If $\mathbb{V}=T X$ and $v$ is a holomorphic Killing vector field, (3-4) is a special case of [Bismut 1992, Theorems 6.2 and 7.7]. In this case, $h_{1}^{\mathbb{V}}=g^{T X}$, and on $Y$, we have a holomorphic and orthogonal splitting $\left.T X\right|_{Y}=T Y \oplus N$. Thus $\widetilde{\mathrm{Td}}\left(T Y,\left.T X\right|_{Y}, g^{\left.T X\right|_{Y}}\right)=0$. To compute $\widetilde{\mathrm{T}}^{-1}\left(N, g^{N}, g_{1}^{N}\right)$, note that $g_{1}^{N}=$ $g^{N}((\nabla v) \cdot,(\nabla v) \cdot)$, as $A=(\nabla v)^{*}(\nabla v)$ is positive and self-adjoint; thus $(A)^{s}$ is well defined for $s \in[0,1]$. Taking $g_{s}^{N}=g^{N}\left((A)^{s} \cdot, \cdot\right)$, we obtain by [Bismut et al. 1988a, Theorem 1.30]

$$
\operatorname{T\widetilde {d}}^{-1}\left(N, g^{N}, g_{1}^{N}\right)=\int_{0}^{1}\left\langle\left(\operatorname{Td}^{-1}\right)^{\prime}\left(N, g_{s}^{N}\right), \log A\right\rangle d s .
$$

But $\nabla v$ is holomorphic, so the curvature $R_{s}^{N}$ associated to the holomorphic connection on $\left(N, g_{s}^{N}\right)$ is $R_{s}^{N}=R^{N}$ for $s \in[0,1]$. Thus

$$
\mathrm{T}^{-1}\left(N, g^{N}, g_{1}^{N}\right)=\left\langle\left(\mathrm{Td}^{-1}\right)^{\prime}\left(N, g^{N}\right), \log A\right\rangle .
$$

Now

$$
\operatorname{Td}\left(T X, g^{T X}\right) T\left(\wedge\left(T^{*} X\right), h^{\wedge\left(T^{*} X\right)}\right)=\tilde{c}_{\max }\left(T X, g^{T X}\right)
$$

is an $(n-1, n-1)$-form on $X$.

In this case, we get easily the special case of [Bismut 2004, Theorem 4.15] directly from [Ray and Singer 1973] by using Poincaré duality:

$$
\frac{\partial \theta^{Y}}{\partial s}(0)=0
$$

From (3-4), (3-6), (3-7), and the vanishing of the constant terms of $R(N)$ and $\frac{\mathrm{Td}^{\prime}}{\mathrm{Td}}\left(N, g^{N}\right)-\frac{1}{2}$, we get

$$
-\frac{\partial \theta_{v, 1}^{X}}{\partial s}(0)=\int_{Y} c_{\max }(T Y)\left(R(N)-\left\langle\frac{\mathrm{Td}^{\prime}}{\mathrm{Td}}\left(N, g^{N}\right)-\frac{1}{2}, \log A\right\rangle\right)=0 .
$$




\section{Appendix: six intermediate results}

In this section, to help readers understand how to obtain Theorem 2.2, we write down the corresponding intermediate results from [Bismut and Lebeau 1991, Theorems 6.4-6.9].

Let $\nabla^{\wedge\left(\mathbb{V}^{*}\right)}$ be the connection on $\wedge\left(\mathbb{V}^{*}\right)$ induced by $\nabla^{\mathbb{V}^{*}}$. Set $C_{u}=\nabla^{\wedge}\left(\mathbb{V}^{*}\right)+$ $\sqrt{u} V$. Let $\mathscr{B}_{T^{2}}^{2}$ and $\operatorname{Tr}_{s}\left(N_{H}^{Y} \exp \left(-\mathscr{P}_{T^{2}}^{2}\right)\right)$ be the operator and the generalized trace associated to the complex (2-7) as in [Bismut and Lebeau 1991, §5]. Let $\Phi$ be the homomorphism from $\wedge^{\text {even }}\left(T_{\mathbb{R}}^{*} X\right)$ into itself which to $\alpha \in \wedge^{2 p}\left(T_{\mathbb{R}}^{*} X\right)$ associates $(2 \pi i)^{-p} \alpha$.

Theorem 4.1. For any $u_{0}>0$, there exists $C>0$ such that for $u \geq u_{0}, T \geq 1$,

$$
\begin{array}{r}
\left|\operatorname{Tr}_{s}\left(N_{H}^{X} e^{-u\left(D^{X}+T V\right)^{2}}\right)-\operatorname{Tr}_{s}\left(\left(\frac{1}{2} \operatorname{dim} N+N_{H}^{Y}\right) e^{-u D^{Y, 2}}\right)\right| \leq \frac{C}{\sqrt{T}}, \\
\left|\operatorname{Tr}_{s}\left(\left(N^{X}-N_{H}^{X}\right) e^{-u\left(D^{X}+T V\right)^{2}}\right)-\operatorname{Tr}_{s}\left(\left(N^{Y}-N_{H}^{Y}\right) e^{-u D^{Y, 2}}\right)\right| \leq \frac{C}{\sqrt{T}} .
\end{array}
$$

Theorem 4.2. Let $\tilde{P}_{T}$ be the orthogonal projection operator from $\Omega\left(X, \wedge\left(\mathbb{V}^{*}\right) \otimes \xi\right)$ to $\operatorname{Ker}\left(D^{X}+T V\right)$. There exist $c>0$ and $C>0$ such that, for any $u \geq 1$ and $T \geq 1$,

$$
\left|\operatorname{Tr}_{s}\left(\left(N^{X}-N_{H}^{X}\right) e^{-u\left(D^{X}+T V\right)^{2}}\right)-\operatorname{Tr}_{s}\left(\left(N^{X}-N_{H}^{X}\right) \tilde{P}_{T}\right)\right| \leq c e^{-C u},
$$

Theorem 4.3. There exist $C>0$ and $\gamma \in] 0,1]$ such that, for any $u \in] 0,1]$ and $0 \leq T \leq 1 / u$,

$$
\left|\operatorname{Tr}_{S}\left(N_{H}^{X} e^{-\left(u D^{X}+T V\right)^{2}}\right)-\int_{X} \operatorname{Td}\left(T X, g^{T X}\right) \Phi \operatorname{Tr}_{S}\left(N_{H}^{X} e^{-C_{T^{2}}^{2}}\right)\right| \leq C(u(1+T))^{\gamma} .
$$

There exists a constant $C^{\prime}>0$ such that for $\left.\left.u \in\right] 0,1\right]$ and $0 \leq T \leq 1$,

$$
\left|\operatorname{Tr}_{s}\left(N_{H}^{X} e^{-\left(u D^{X}+T V\right)^{2}}\right)-\operatorname{Tr}_{s}\left(N_{H}^{X} e^{-\left(u D^{X}\right)^{2}}\right)\right| \leq C^{\prime} T .
$$

Theorem 4.4. For any $T>0$,

$\lim _{u \rightarrow 0} \operatorname{Tr}_{s}\left(N_{H}^{X} e^{-\left(u D^{X}+(T / u) V\right)^{2}}\right)=\int_{Y} \Phi \operatorname{Tr}_{s}\left(N_{H}^{Y} e^{-\mathscr{B}_{T}^{2}}\right) \operatorname{ch}\left(\wedge\left(\mathbb{V}_{1}^{*}\right), h^{\wedge\left(\mathbb{V}_{1}^{*}\right)}\right) \operatorname{ch}\left(\xi, h^{\xi}\right)$.

Theorem 4.5. There exist $C>0$ and $\delta \in] 0,1]$ such that, for any $u \in] 0,1]$ and $T \geq 1$,

$$
\left|\operatorname{Tr}_{s}\left(N_{H}^{X} e^{-\left(u D^{X}+(T / u) V\right)^{2}}\right)-\operatorname{Tr}_{s}\left(\left(\frac{1}{2} \operatorname{dim} N+N_{H}^{Y}\right) e^{-u D^{Y, 2}}\right)\right| \leq \frac{C}{T^{\delta}} .
$$

Let $|\cdot|_{\lambda_{v}\left(\mathbb{V}^{*}\right), T}^{2}$ be the $L^{2}$-metric on $\lambda_{v}\left(\mathbb{V}^{*}\right)$ induced by $g^{T X}, T^{2} h^{\mathbb{V}}$ as in (2-5). 
Theorem 4.6. As $T \rightarrow+\infty$,

$\log \left(\frac{|\cdot|_{\lambda_{v}\left(\mathbb{V}^{*}\right), T}^{2}}{|\cdot|_{\lambda_{v}\left(\mathbb{V}^{*}\right)}^{2}}\right)$
$\quad=-\log |\rho|_{\lambda\left(\mathbb{V}_{1}^{*}\right)^{-1} \otimes \lambda_{v}\left(\mathbb{V}^{*}\right)}^{2}+\operatorname{Tr}_{s}\left(\left(\operatorname{dim} N+2 N_{H}^{Y}\right) Q\right) \log T+O\left(\frac{1}{T}\right)$.

\section{Acknowledgements}

Feng thanks Jean-Pierre Bourguignon and the IHES, where part of this research was performed, for their hospitality. Thanks also to K. Liu for drawing our attention to [Beasley and Witten 2003].

\section{References}

[Beasley and Witten 2003] C. Beasley and E. Witten, "Residues and world-sheet instantons", 2003. hep-th/0304115

[Bershadsky et al. 1993] M. Bershadsky, S. Cecotti, H. Ooguri, and C. Vafa, "Holomorphic anomalies in topological field theories", Nuclear Phys. B 405:2-3 (1993), 279-304. MR 94j:81254 Zbl 1039.81550

[Bershadsky et al. 1994] M. Bershadsky, S. Cecotti, H. Ooguri, and C. Vafa, "Kodaira-Spencer theory of gravity and exact results for quantum string amplitudes", Comm. Math. Phys. 165:2 (1994), 311-427. MR 95f:32029 Zbl 0815.53082

[Bismut 1986] J.-M. Bismut, "Localization formulas, superconnections, and the index theorem for families", Comm. Math. Phys. 103:1 (1986), 127-166. MR 87f:58147 Zbl 0602.58042

[Bismut 1992] J.-M. Bismut, "Bott-Chern currents, excess normal bundles and the Chern character", Geom. Funct. Anal. 2:3 (1992), 285-340. MR 94a:58206 Zbl 0776.32007

[Bismut 2004] J.-M. Bismut, "Holomorphic and de Rham torsion", Compos. Math. 140:5 (2004), 1302-1356. MR 2081158 Zbl 02110378

[Bismut and Lebeau 1991] J.-M. Bismut and G. Lebeau, "Complex immersions and Quillen metrics”, Inst. Hautes Études Sci. Publ. Math. 74 (1991), 1-297. MR 94a:58205 Zbl 0784.32010

[Bismut et al. 1988a] J.-M. Bismut, H. Gillet, and C. Soulé, "Analytic torsion and holomorphic determinant bundles, I: Bott-Chern forms and analytic torsion", Comm. Math. Phys. 115:1 (1988), 49-78. MR 89g:58192a Zbl 0651.32017

[Bismut et al. 1988b] J.-M. Bismut, H. Gillet, and C. Soulé, "Analytic torsion and holomorphic determinant bundles, III: Quillen metrics on holomorphic determinants", Comm. Math. Phys. 115:2 (1988), 301-351. MR 89g:58192c Zbl 0651.32017

[Bismut et al. 1990] J.-M. Bismut, H. Gillet, and C. Soulé, "Complex immersions and Arakelov geometry”, pp. 249-331 in The Grothendieck Festschrift, vol. I, Progr. Math. 86, Birkhäuser, Boston, 1990. MR 92a:14019 Zbl 0744.14015

[Bott 1967] R. Bott, “A residue formula for holomorphic vector-fields", J. Differential Geometry 1 (1967), 311-330. MR 38 \#730 Zbl 0179.28801

[Feng 2003] H. Feng, "Holomorphic equivariant cohomology via a transversal holomorphic vector field”, Internat. J. Math. 14:5 (2003), 499-514. MR 2004j:32022 Zbl 1050.32013

[Gillet and Soulé 1991] H. Gillet and C. Soulé, "Analytic torsion and the arithmetic Todd genus", Topology 30:1 (1991), 21-54. MR 92d:14015 Zbl 0787.14005 
[Liu 1995] K. Liu, "Holomorphic equivariant cohomology", Math. Ann. 303:1 (1995), 125-148. MR 97f:32041 Zbl 0835.14006

[Ray and Singer 1973] D. B. Ray and I. M. Singer, "Analytic torsion for complex manifolds", Ann. of Math. (2) 98 (1973), 154-177. MR 52 \#4344 Zbl 0267.32014

[Zhang 1990] W. Zhang, "A remark on a residue formula of Bott", Acta Math. Sinica (N.S.) 6:4 (1990), 306-314. MR 91j:58153 Zbl 0738.32007

[Zhang n.d.] W. Zhang, "Equivariant Dolbeault complex and total Quillen metrics", preprint.

Received July 25, 2003. Revised February 13, 2004.

Huitao Feng

College of Mathematical Sciences

NANKAI UNIVERSITY

300071, TIANJIN

CHINA

fht@nankai.edu.cn

XiaOnan Ma

CEnTRE DE MATHÉmatiQues

UMR 7640 DU CNRS

ÉCOLE POLYTECHNIQUE

91128 Palaiseau CedeX

FRANCE

ma@math.polytechnique.fr 